\title{
Breastfeeding Practices Among Women in Delhi: A Cohort Study
}

\author{
Agrawal S, Monika M, Pathak P, Kaur H, Kanika Chopra* and Trivedi SS \\ Department of Obstetrics and Gynecology, India
}

ISSN: 2640-9666

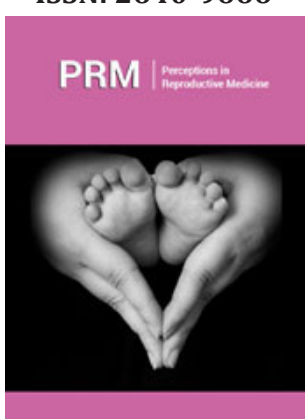

*Corresponding author: Kanika Chopra, Department of Obstetrics and Gynecology, Lady Hardinge Medical College, New Delhi, India

Submission: 眥June 06, 2019

Published: 踖July 03, 2019

Volume 3 - Issue 3

How to cite this article: Agrawal S, Monika M, Pathak P, Kaur H, Kanika Chopra, et al. Breastfeeding Practices Among Women in Delhi: A Cohort Study. Perception in Reproductive Medicine.3(3). PRM.000564.2019.

DOI: 10.31031/PRM.2019.03.000564

Copyright@ Kanika Chopra, This article is distributed under the terms of the Creative Commons Attribution 4.0 International License, which permits unrestricted use and redistribution provided that the original author and source are credited.

\begin{abstract}
Introduction: WHO recommends that all infants should be exclusively breastfed for six months after birth. However, practices in India are largely influenced by traditional family practices, knowledge and attitude towards breastfeeding among the population and this study was planned to assess factors associated with breastfeeding among women in Delhi.
\end{abstract}

Material and Methods: A total of two hundred eight antenatal women attending the outpatient department of a tertiary care centre in Delhi were randomly selected and interviewed. Information regarding participants demographic profile, their knowledge and attitude towards breastfeeding and infant feeding in the first 6 months was collected.

Results: In the study, $81 \%$ of women were aware of the beneficial effects of breastfeeding for both the mother and the child. Also, $80 \%$ were in favour of continuing it for 6 months and $75 \%$ marked their affirmation to start weaning thereafter. However, incorrect practices of giving pre-lacteal feed and bottle feeding were practiced by nearly one-third of the mothers. Fifty percent of women felt that their knowledge and awareness about breastfeeding is inadequate and that more information is needed.

Conclusion: Majority of respondents in this study had a positive attitude towards breastfeeding but incorrect practices like giving ghutti, honey etc and bottle feeding of the baby were accepted and practiced by nearly one third of women. There is a need to reinforce the advantages of breastfeeding in the community with an emphasis to avoid prelacteal feed.

\section{Introduction}

A healthy start in life is the most precious gift one can give to a newborn child. Breast feeding is said to be the best food for an infant and has innumerable benefits for both the mother and the child. WHO and UNICEF recommend that exclusive breast feeding should be the norm for the first 4-6 months and breast feeding should be continued together with weaning food up to and beyond second year of life [1]? However, it has been seen that breast-feeding practices especially exclusive breast feeding is on a decline worldwide owing to multiple reasons with lack of awareness being the most important cause. In India, age old family tradition influence breast feeding practices a lot and thus this leads to under nutrition, malnutrition, infections and ultimately to infant mortality. This present study was thus planned to understand the knowledge, attitude and practice of the mothers under study, so that appropriate steps can be taken to inculcate healthy breastfeeding practices in the population.

\section{Material and Methods}

Our study was a prospective study, carried out in the Department of obstetrics and gynecology, Lady Hardinge Medical College, New Delhi, over a period of 6 months and included two hundred eighty-six mothers who utilized our obstetrics services. All the participating mothers were given questionnaire with multiple choice questions in their native language (Hindi). The questionnaire was directed to comprehend certain particulars of the women such as age, education, socioeconomic status, occupation, practices of breast feeding and awareness among them and they were told to mark the most appropriate choice as per their practice and knowledge.

\section{Result}

In our study, most mothers (58.74\%) were between the ages of 26-30 years of age and average age of the study population was 24.29 years. Among the age group of 16-20 years, 
21-25 years, 31-35 years and more than 35 years, there were $12.9 \%, 24.8 \%, 3.14 \%$ and $0.34 \%$ mothers respectively. Most (94\%) of the mothers were literate with $18 \%$ having education status of graduate and above. Fifteen mothers among 286 were illiterate (5.24\%) but were able to understand the questionnaire when read out by the interviewer and gave their responses. Majority mothers i.e. $48.2 \%$ belonged to low income group <Rs $4000 /$ month and were housewives. It was revealed that $81 \%$ of women were aware of the beneficial effects of breastfeeding for both the mother and the child. It was deciphered that $71 \%$ mothers felt the need of initiating breast feeding immediately after birth and 65\% were aware of the importance of initial milk i.e. colostrum and knew that it should not be discarded. Further, $80 \%$ were in favor of continuing breastfeeding for 6 months and $75 \%$ marked their affirmation to start weaning thereafter. Majority mothers $(80 \%)$ said that they had no difficulty in initiating breast feeding and also felt that their feed was adequate for the baby.

Among two hundred eighty-six mothers; to our surprise, only 12 mothers said that exclusive breast feeding should be continued for 6 months accounting to just 4\%. Taking into consideration, pre-lacteal feed, $30 \%$ responded that ghutti or honey should be given at birth, $58 \%$ said that water should be given before 6 months and $35 \%$ were in favor of giving bottle feed instead of breast feed. The principal source of information regarding breast feeding was doctors/health staff in $56 \%$, followed by mothers and family and friends in $40 \%$. However, $50 \%$ of women still felt that their knowledge and awareness about breastfeeding is inadequate and that more information is needed.

\section{Discussion}

Despite the nutritional, immunological, physiological and psychological benefits of breast feeding for both the mother and the child, its importance is on rapid decline in both developed and developing countries due to innumerable reasons. This paper presents finding of a study in which 286 mothers were interviewed and their knowledge, attitude and practice of breast feeding assessed. The increase in the use of formula feed or cow's milk in an assumption that mother's milk is inadequate and giving water prior to 6 months to ensure baby is not thirsty are solely due to lack of awareness. Colostrum, which is known to be the most nutritious part of breast milk with immunological benefits, is secreted in the first 48-72 hours before changing to breast milk and is usually discarded. This is rampant in areas of Rajasthan, India [2]. Bhavna Singh in her study revealed that the majority of the mothers interviewed were of the age group of $21-30$ years i.e. around $76.07 \%$ comparative to our study. Among them, $17 \%$ had acquired high school education and above and $17 \%$ had no education but were able to understand the questions and $28 \%$ belong to low income group. It was found in this study that only $7 \%$ mothers were of the opinion of giving water after 6 months, negating the importance of exclusive breast feeding for 6 months compared to our study [3]. In another study from Bangladesh by Haider et al. [4], prevalence of mothers practicing recommended behavior was evaluated. Breast feeding initiation within first hour of birth was recommended by $24 \%$, giving colostrum by $46 \%$, not bottle feeding by $85 \%$ and exclusive breast feeding by $10 \%$. The most reliable source of information was grandmother in $28 \%$ followed by doctors in $24 \%$. Grover et al. [5] in their study on knowledge, attitude and practice on breast feeding in rural areas of East Delhi evaluated that 71\% mothers agreed that breast feeding is the healthiest food for infants. However, prelacteal feed was given by $82 \%$ mothers which included ghutti and honey as in our study but was comparatively much high.

The present study reveals that although our study population had low levels of education and belonged to low income group but, still they were well aware about immediate starting of breastfeeding after birth, exclusive breast feeding although in minority, weaning after six months and beneficial effects of breastfeeding for both the mothers and the child. Easy and prompt access to information about breast feeding practices via health personals and media in Delhi, India led to high awareness about correct breast-feeding knowledge. However, incorrect practices of giving pre-lacteal feeds, giving water before 6 months and bottle feeding the babies also exists and needs addressing of this issue at national level. Imparting knowledge to mothers should be made a primary focus during antenatal checkups and post-delivery, there should be allotment of special health staff who will help the mother initiate early breast feeding, explain how to lactate properly and emphasis on avoiding any other food other that breast feed for infants less than 6 months. At every visit of mother for immunization of her child, there should be availability of health personals that can ensure exclusive breast feeding and give proper information about weaning.

\section{Conclusion}

Nursing is not only nutritionally beneficial to the baby, but also it is the safest form of feed that can be given to the baby without the risk of contamination. It is economical as well, which is of major concern in a resource poor country. There should be strong emphasis on training of health staff dealing with antenatal patients and mothers of infants to educate them about the innumerable benefits of breastfeeding. There should be combined efforts of both the government and the non-government association to design policies, like limiting advertisements of infant feeding formula, taking help from media to accentuate the importance of breastfeeding and promoting messages like early onset of breastfeeding after delivery, giving colostrum and exclusive breast feeding for 6 months that does not include even water. Special trainers explaining the proper method of latching should be appointed in all set ups and also clinics that can cater to any breast-feeding related issues. All these efforts will help in developing a healthy future by raising healthy infants.

\section{References}

1. (1992) Take the baby friendly initiative. UNICEF, New York, USA.

2. Singh MB, Haldiya KR, Laxminarayan J (1997) Infant feeding and weaning practices in some semi-arid rural areas of Rajasthan. Journal Indian Med Assoc 95(11): 576-578.

3. Singh B (2010) Knowledge, attitude and practice of breast feeding-A case study. European Journal of Scientific Research 40(3): 404-422. 
4. Haider R, Rasheed S, Sanghvi TG, Hassan N, Pachon H, et al. (2010) Breastfeeding in infancy: identifying the program-relevant issues in Bangladesh. Int Breastfeed J 5: 21.
5. Grover VL, Chhabra P, Aggarwal OP (1997) Knowledge, attitude and practices of breast feeding in a rural area of East Delhi. Health and Population-Perspectives and Issues 20(2): 49-56.

For possible submissions Click below: 